San Jose State University

SJSU ScholarWorks

Master's Projects

Master's Theses and Graduate Research

Spring 2019

\title{
An Industry Driven Genre Classification Application using Natural Language Processing
}

Sharan Duggirala

San Jose State University

Follow this and additional works at: https://scholarworks.sjsu.edu/etd_projects

Part of the Computer Sciences Commons

\section{Recommended Citation}

Duggirala, Sharan, "An Industry Driven Genre Classification Application using Natural Language

Processing" (2019). Master's Projects. 737.

DOI: https://doi.org/10.31979/etd.gyyw-7de5

https://scholarworks.sjsu.edu/etd_projects/737

This Master's Project is brought to you for free and open access by the Master's Theses and Graduate Research at SJSU ScholarWorks. It has been accepted for inclusion in Master's Projects by an authorized administrator of SJSU ScholarWorks. For more information, please contact scholarworks@sjsu.edu. 


\title{
An Industry Driven Genre Classification Application using Natural Language Processing
}

\author{
by \\ Sharan Duggirala
}

A dissertation submitted in partial satisfaction of the

requirements for the degree of

Masters

in

Computer Science

in the

Graduate Division

of the

San Jose State University

Committee in charge:

Professor Teng Moh, Chair

Professor Chris Pollett

Mr. Kevin Smith

Spring 2019 
The dissertation of Sharan Duggirala, titled An Industry Driven Genre Classification Application using Natural Language Processing, is approved:

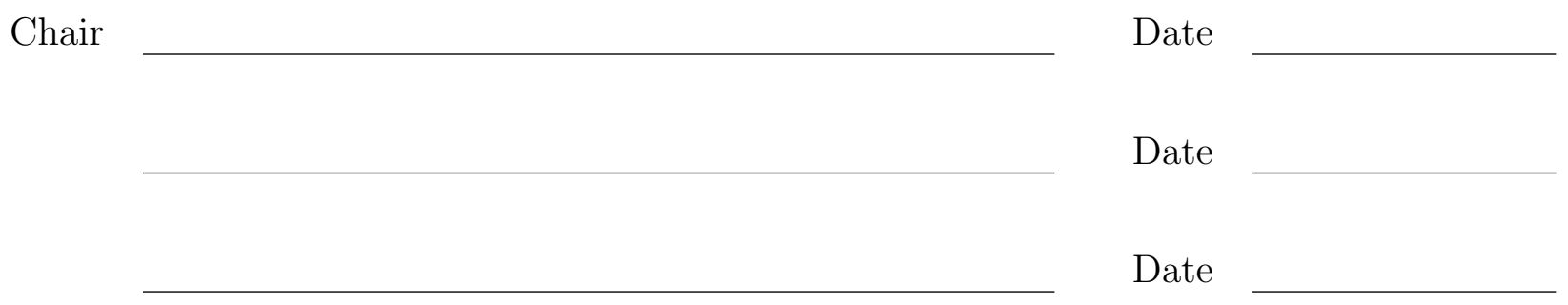

San Jose State University 
An Industry Driven Genre Classification Application using Natural Language

\section{Processing}

Copyright 2019

by

Sharan Duggirala 


\begin{abstract}
by

Sharan Duggirala

Masters in Computer Science

San Jose State University

Professor Teng Moh, Chair
\end{abstract}

An Industry Driven Genre Classification Application using Natural Language Processing

With the advent of digitized music, many online streaming companies such as Spotify have capitalized on a listener's need for a common stream platform. An essential component of such a platform is the recommender systems that suggest to the constituent user base, related tracks, albums and artists. In order to sustain such a recommender system, labeling data to indicate which genre it belongs to is essential. Most recent academic publications that deal with music genre classification focus on the use of deep neural networks developed and applied within the music genre classification domain. This thesis attempts to use some of the highly sophisticated techniques, such as Hierarchical Attention Networks that exist within the text classification domain in order to classify tracks of different genres. In order to do this, the music is first separated into different tracks (drums, vocals, bass and accompa- 
niment) and converted into symbolic text data. Due to the sophistication of the distributed machine learning system (over five computers, each possessing a graphical processing units greater than a GTX 1070) present in this thesis, it is capable of classifying contemporary genres with an impressive peak accuracy of over 93\%, when comparing the results with that of competing classifiers. It is also argued that through the use text classification, the expert domain knowledge which musicians and people involved with musicological techniques, can be attracted to improving reccomender systems within the music information retrieval research domain. 
To all the people that have supported me throughout this entire project. 


\section{Contents}

$\begin{array}{ll}\text { Contents } & \text { ii }\end{array}$

List of Figures $\quad$ iv

List of Tables $\quad$ v

1 Introduction $\quad 1$

2 Related Works 3

2.1 Traditional Classification . . . . . . . . . . . . . . . . . . 3

2.2 Natural Language Proceessing and Word2Vec . . . . . . . . . . . . . . . . 5

2.3 Deep Learning Classification . . . . . . . . . . . . . . . . . . . . . . 11

2.4 Hierarchical Attention Networks . . . . . . . . . . . . . . . . . 14

3 Proposed Method $\quad 17$

3.1 Feature Extraction . . . . . . . . . . . . . . . . . . . 18

3.2 Conversion of Melodic Data into Symbolic Data . . . . . . . . . . . . . . 22

3.3 Intelligent Phrase Separation and Append Operator . . . . . . . . . . . . . . 24

3.4 Creating the word Embeddings . . . . . . . . . . . . . . . 26

3.5 Calculating Sentence Length . . . . . . . . . . . . . . . . . . . 27

3.6 Classification Pipeline . . . . . . . . . . . . . . . . . 28

4 Implementation Deatils $\quad 32$

4.1 Dataset . . . . . . . . . . . . . . . . . . . 32

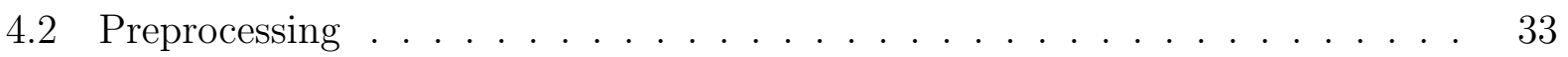

4.3 Testing and deep learning frameworks . . . . . . . . . . . . . . . . . . . . . . . . . . . . . . .

4.4 Distributed Learning . . . . . . . . . . . . . . . . . . . . . . . . . . 34

5 Results $\quad 36$

6 Conclusion $\quad 40$

6.1 Conclusion . . . . . . . . . . . . . . . . . . 40 
6.2 Future Works . . . . . . . . . . . . . . . . . . . . . . . 41

$\begin{array}{ll}\text { Bibliography } & 42\end{array}$ 


\section{List of Figures}

2.1 A simple CNN that creates a matrix representation of the sentence and proceeds to try out multiple convolutional filters, each of a different size on the matrix, followed by a max pooling operator. The result of this operation is the input to a fully connected layer which results in a classification of the input sentence. [1]

2.2 The architecture of a simple RNN. This is the unrolled version of an RNN as indicated by the unrolled network on the right hand side and the actual network diagram on the left hand side $[2] \ldots \ldots$. . . . . . . . . . . . .

2.3 the internals of an attention mechanism that will be used in this thesis. Each of the red hidden states have received a lot more of the distributed attention over the blue hidden states $[1] . \ldots \ldots \ldots$

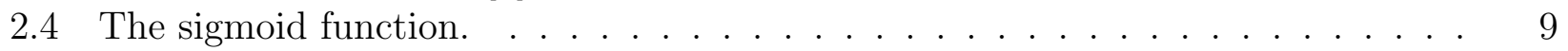

2.5 The ReLU function. . . . . . . . . . . . . . . . . . . . . . . . . . . . . 10

2.6 AUCs for the models that were mentioned in [3]. The number of parameters indicates the total number of the training parameters within the model, and how its complexity scales. . . . . . . . . . . . . . . . . . . . . . . . . . . . . . . . . . . . . .

2.7 A heirarchical attention network. . . . . . . . . . . . . . . 16

3.1 The outline of the architecture being proposed by this thesis. . . . . . . . . . 17

3.2 The feature extraction architecture being proposed by this thesis. . . . . . . . . 18

3.3 Drum Transcription Neural Network . . . . . . . . . . . . . . . . . . . 19

3.4 Architecture of the Track Separation Neural Network . . . . . . . . . . . . . . . 21

3.5 Modified HAN that's being used for the classification pipeline of this publication. 29

5.1 The AUC ROC graph as per the different genres. . . . . . . . . . . . . . . . 39 


\section{List of Tables}

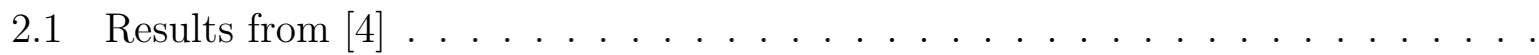

4.1 Breakdown of the number of tracks per genre on the GTZAN[5] and ISMIR[6] datasets . . . . . . . . . . . . . . . . . . .

4.2 The specifications of the experimental setup. it must be noted that testing took place on a single computer, which was the master and also the system with the largest GPU memory available. . . . . . . . . . . . . . . . . . . . .

5.1 The performance of different combinations of genres and their respective F1 score rounded to two significant figures. . . . . . . . . . . . . . . . . .

5.2 The performance of the proposed solution with different combinations of preprocessed features . . . . . . . . . . . . . . . . . . 


\section{Acknowledgments}

I would like to acknowledge Anusha Sridharan for her help with my coding of the symbolic text conversion. I would like to thank Siddharth Kulkarni and Michael Symmonds for their contributions to the modifications of the hyper parameters of the text classification deep learning algorithms. Charles MacKay was a constant guide and mentor through the use of various techniques throughout the development process. Finally Revanth Akella deserves a mention as he was a constant inspiration in terms of the different techniques that were used. 


\section{Chapter 1}

\section{Introduction}

Digital music is an incredibly large industry that has a special relationship with academia through Music Information Retrieval (MIR). Both Digital Platform companies such as Spotify and the MIR research communities are trying to improve the personalization of these digitized systems. In order to do so, retrieval and recommender systems are necessary, which both use Music genre classification as a core component.

Many contemporary techniques for music genre classification have been researched. Zheng, Moh, Moh [4] indicate that there are three different descriptors that can be used for building such systems: descriptive metadata, low level audio features, high level music content description. Descriptive metadata is pure text containing information about a certain piece of music, that has been created by a human being. Some of the disadvantages of such data is the likelihood that it is false(improper tagging), and the lack of a enforced standardized protocol to such data. Low level audio features can be extracted from the audio file itself 
and is often very efficient as a feature. However, this data is not interpretable to dilettantes or experts within the music field. High level descriptors overcome this problem by being interpretable to music aficionados and musicians themselves, thus utilize the intuitions and domain knowledge of aforementioned people.

Historically, a lot of different research publications have used the descriptive metadata or the low level audio features in order to classify tracks. This is mostly due to the ease of which, features for machine learning, can be extracted and transformed, with respect to low level feeatures. Very few publications explore the usage of high level descriptors such as symbolic text data to classify an audio file into a particular genre. No publications have approached the genre classification problem domain using the text classifiers that have been developed within the Natural Language Processing (NLP) field that has seen much increase and growth over the last few years, due to the renaissance of deep learning.

The rest of this report is written in the sections as follows. Chapter 2 looks at previous attempts at music classification and the pertinent results. This section is divided into multiple subsections that deal with introducing music through traditional Machine Learning, Deep Neural Networks, symbolic text data, Word2Vec and finally text classification that is an essential part of Natural Language Processing. Chapter 3 expands on the proposed method, while Chapter 4 addresses the experiments and results. Chapter 5 expounds the conclusion from the results and the findings of the paper. It also expands on the different areas and works that could not be tackled within the scope of this research work. 


\section{Chapter 2}

\section{Related Works}

\subsection{Traditional Classification}

One of the most intuitive ways to approach classifying music, would be to measure the similarity between two records, one of which is labeled. This similarity can be considered as a distance value that is computed between a features. Algorithms such as K-Nearest Neighbor have been used by M. Li, R.Sleep [7]. Kotsifakos et. al [8], have built their own classifier based on a similarity function built specially for music genre classification. One major disadvantage of such a technique is inherited from the music information retrieval system from which it is adapted from. Genre classification would require an increasing number of features, increasing the dimensionality of the data (this would render techniques such as clustering at a disadvantage) making such classification techniques not interpretable to experts. Techniques derived from well performed music information retrieval systems 


\begin{tabular}{|l|l|l|l|l|l|}
\hline \multicolumn{2}{|l|}{ Part Feature } & melody & bass & melody+bass & bass+melody \\
\hline \multirow{4}{*}{ N-gram range } & $(1,1)$ & 0.75 & 0.85 & 0.86 & 0.86 \\
\cline { 2 - 6 } & $(2,2)$ & 0.84 & 0.89 & 0.91 & 0.91 \\
\cline { 2 - 6 } & $(3,3)$ & 00.82 & 0.92 & 0.92 & 0.93 \\
\cline { 2 - 6 } & $(1,2)$ & 0.82 & 0.98 & 0.98 & 0.98 \\
\cline { 2 - 6 } & $(1,3)$ & 0.81 & 0.91 & 0.92 & 0.92 \\
\hline
\end{tabular}

Table 2.1: Results from [4]

don't guarantee performance, as Kotsifakos et. al's work's accuracy lies between 30\%-40\%.

Symbolic data is a novel approach within the music genre classification sphere. McKay and Fujinaga [9] were one of the first to analyze statistical distributions of global feature sets extracted, from MIDI data, through $j M I R$, a platform created by the aforementioned researches to extract high level features and classify the MIDI file through machine learning functionalities. The dataset used within the paper contained three root sub genres and nine sub-genres. While the platform performed well with multi-instrumental data sources, it only looks at global statistical data and therefore lacks any intuitive support from experts.

The most recent efforts in terms of symbolic data classification within the Music Information Retrieval is Zheng, Moh, Moh[4] attempt to classify musical genres through an N-gram based musicological approach which achieved a prediction accuracy over $90 \%$ on an average. The 5-fold cross validation accuracy with a plethora of different features used by Zheng, Moh, Moh is described in Table 2.1. 


\subsection{Natural Language Proceessing and Word2Vec}

Natural language processing (NLP) is a combination of computer science, information engineering and artificial intelligence that studies the interaction between computers and human natural languages. This often involves studying how computational algorithms can analyze and represent the human language. Some of the most common uses of NLP include search engines and voice activated assistants.

Word2Vec is a set of techniques and models that produce word embeddings. Word embeddings are based on a distributional hypothesis where words appearing within similar context possess similar meaning and therefore share a context. Using a shallow network word embeddings usually predict a word based on its context. Mikolav et al. [10] proposed Word2Vec with 2 different models. Conditional Bag of Words (CBOW) computes the conditional probability of a target based on the context of neighboring in a given window size. Skip-gram also constructs word embeddings, where the conditional probability is that of the surrounding words, given the target word at the center. While Word2Vec is not a supervised learning technique, it is definitely considered self supervised where the Both these techniques use shallow neural networks. Word embeddings have been shown to improve the performance of NLP tasks such as syntactic parsing and sentiment analysis.

A convolutional neural network is a neural based approach that represents a function that is applied to words or n-grams to extract higher level features. In order to provide a sentence to a convolutional operator the sentence is first tokenized into words that undergo 


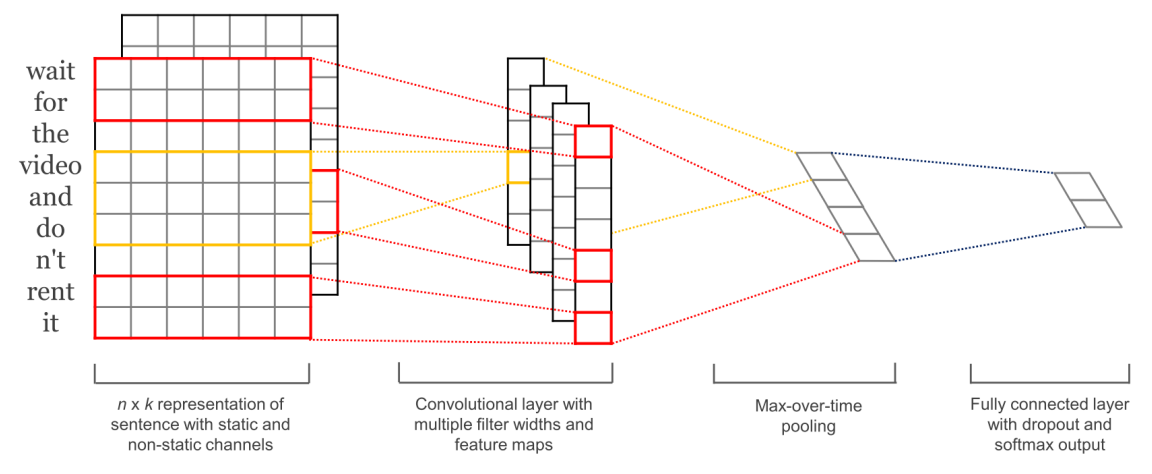

Figure 2.1: A simple CNN that creates a matrix representation of the sentence and proceeds to try out multiple convolutional filters, each of a different size on the matrix, followed by a max pooling operator. The result of this operation is the input to a fully connected layer which results in a classification of the input sentence. [1]

transformation into a word embedding matrix of d dimensions. This enables the convolutional operator to operate on the multi-dimensional matrix and extract features using max pooling. One of the key disadvantages of the convolutional neural networks lie in the fact that long distance sentence dependencies cannot be inferred or learned by CNNs. However, time delayed neural networks (TDNN) proposed by Waibel, Hanazawa, Hinton et. al [11], used extensively in speech recognition and dynamic convolutional neural networks (DCNN) [12]. Generally CNNs struggle at preserving sequential order and long-distance contextual information in pertinence to natural language processing tasks. An example of a simple CNN based text classifier is described in Figure 2.1.

Recurrent neural networks are specialized in processing sequential information and compute every instance of the input sequence conditioned by previous computer result. Recurrent Neural Networks are extensively used and expanded upon in hierarchical attention networks, 


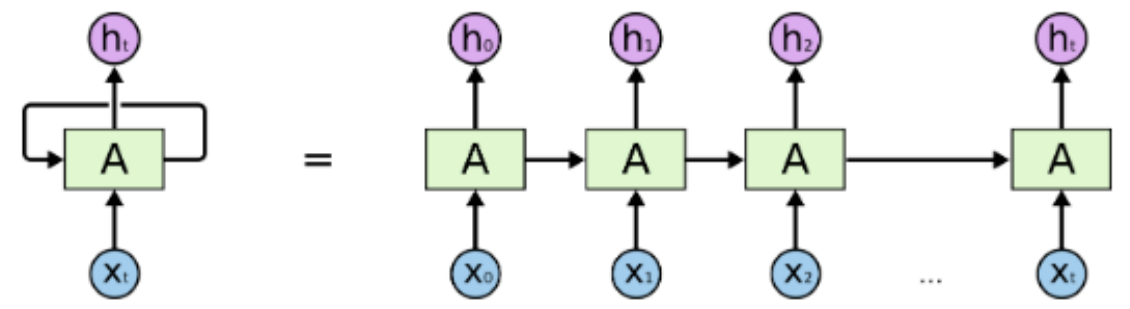

Figure 2.2: The architecture of a simple RNN. This is the unrolled version of an RNN as indicated by the unrolled network on the right hand side and the actual network diagram on the left hand side [2].

described later in this section. Due to the architectural design of the RNNs, RNNs can capture the contextual dependencies of inputs of arbitrary length and therefore are very useful for NLP tasks such as machine translation, image captioning etc. Simple RNNs suffer from the vanishing gradient problem and thus long short term memory (LSTM),residual networks (ResNets) and gated recurrent units (GRUs) were introduced. An LSTM contains there gates, the input, the forget and the output, while the GRUs only contain two (for the sake of efficiency). The architecture of an RNN is best described in Figure 2.2. RNNs have been extensively used in many NLP applications such a word level classification, semantic matching and language modeling etc. In fact, the primary technology of this thesis, Hierarchical Attention Networks apply RNNs practically to solve sentence and document classification.

The encoder-decoder LSTM is an RNN that is designed to address sequence to sequence problems, such as machine translation. Due to the fact that the input and the output of a machine translation application are not fixed length, the encoder encodes the input into a fixed length vector and the decoder decodes this fixed length vector to produce an 


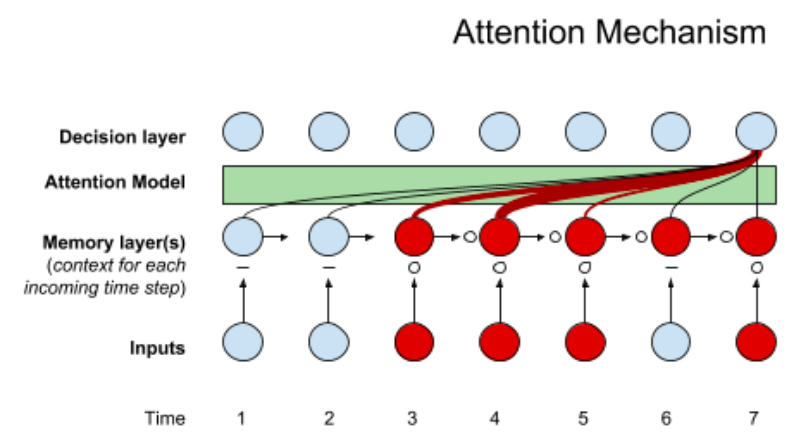

Figure 2.3: the internals of an attention mechanism that will be used in this thesis. Each of the red hidden states have received a lot more of the distributed attention over the blue hidden states [1].

output. Attention Mechanisms are a relatively novel approach to NLP. One of the limits of traditional word vectors is that they presume that every word's meaning is relatively stable across multiple sentences. Another limitation of LSTMS is that the information is outputted from the final hidden state of the network and therefore, the previous states do not have much direct influence on the output of the hidden layer to the decision layer. An attention mechanism helps combat both these issues by taking into account input from multiple hidden state steps through a distributed attention network as shown in Figure 2.3.

Some other common machine learning techniques need to be discussed in order to elaborate on concepts within this thesis. Batch normalization is used extensively in machine learning in order to speed up the amount of time it takes to train a neural network, as normalizing each batch that is an input to the neural network results in dealing with internal covariate shift. The inputs can be standardized through the mean and the standard deviation per batch or a running average across all batches. However, a running average has 


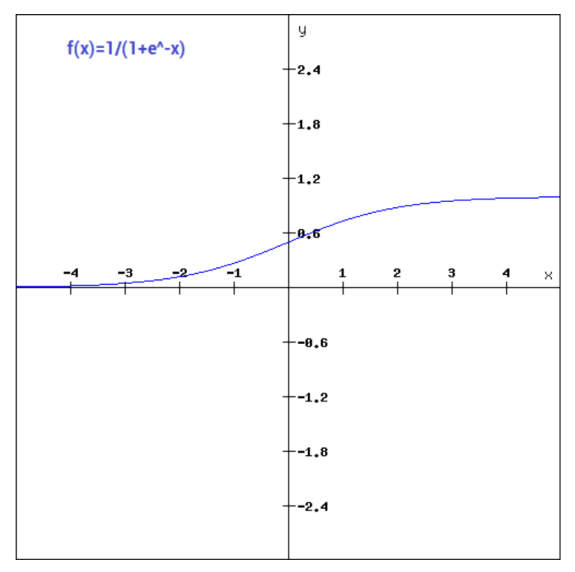

Figure 2.4: The sigmoid function.

been known to sabotage training [?] and thus will be avoided within this thesis. Another aspect of machine learning that must be payed attention to is the different kinds of activation functions. One of them is the different kinds of activation functions. binary, linear and Tanh are avoided, due to there being a trend of decreased use of these activation functions within the Natural Language Processing deep learning communities. However, sigmoid and ReLU have been widely encountered during the literature survey phase of the project outlined in this thesis.

The sigmoid activation function that is most commonly used can be represented by the equation below and the Figure 2.4.

$$
f(x)=\frac{1}{\left(1+e^{-x}\right)}
$$

Small changes in the value of $x$ can bring about great changes to the value of $y$. Due to this ability of the function to push the result to the extreme values of $y$, the sigmoid function 


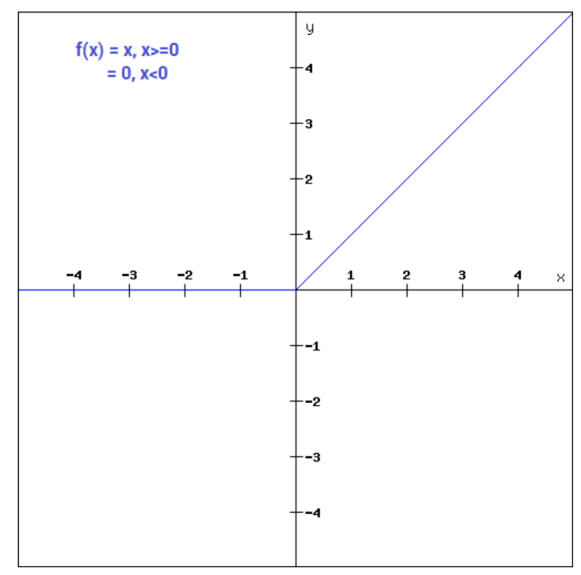

Figure 2.5: The ReLU function.

is particularly useful when trying to classify particular values into classes.

The ReLU activation function can be represented by the equation below and the Figure 2.5. This is the most commonly used activation function as of right now. One issue with ReLU is the fact that all negative values immediately become 0 , thus decreasing the ability of the model to fit the data properly. Leaky ReLU fixes this issue.

$$
f(x)=\max (0, x)
$$

The softmax function which was used in this publication will be elaborated on within the Chapter 4. 


\subsection{Deep Learning Classification}

In this section we examine three techniques used recently in order to classify musical genres. Most of these represent cutting edge models for classification within the academic sphere as of right now.

Choi, Fazekas and Sandler [3], introduce the concept of a convolution recurrent neural (CRNNs) that excels at music genre classification as it uses the CNN for feature extraction and the RNN for temporal summary of the extracted features. Adopting an RNN for aggregating the features enables the network to account for the global structure while local features are extracted by the convolutional layers. The architecture of this model can be best described as a CNN where the last convolutional layers have been replaced with an RNN. This uses a four layer CNN followed by a two layer RNN, the input to which is the 2D kernel of the frequency-time band.

This publication proceeds to compare the performance of the CRNN against three different CNNs with unique architectures that have been inspired by the CNNs being used in the music genre classification domain as of right now. The first one [13], titled $k 1$ consists of 4 convolutional layers that are followed by two connected layers. The second model, titled [14] consists of five convolutional layers and is followed by two fully connected layers. the third model, titled [?][15], also contains five convolutional layers. The key difference between $k 2$ and $k 3$ is the fact that $k 2$ uses a lot of one dimensional convolutional layers, while $k 3$ can be considered fully convolutional as there are stages of gradual 2D sub samplings. 


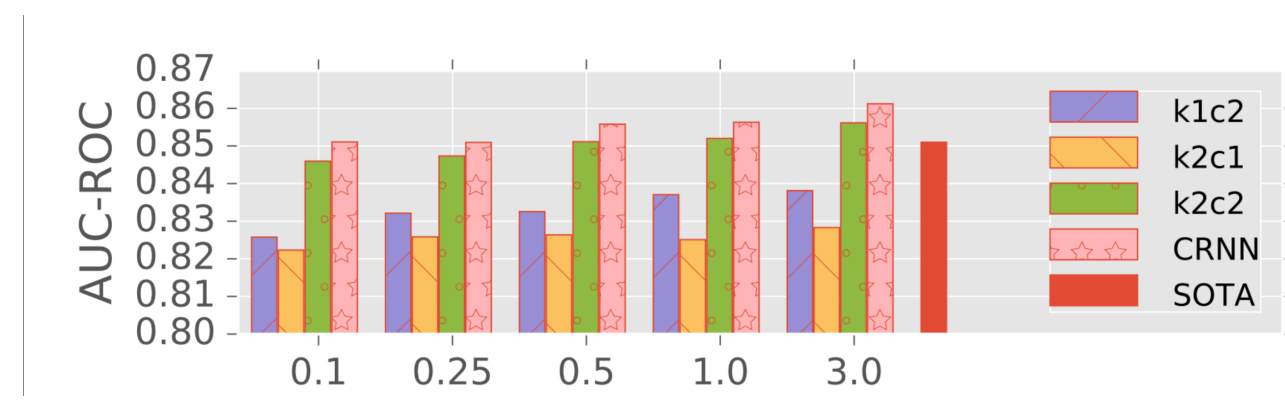

Figure 2.6: AUCs for the models that were mentioned in [3]. The number of parameters indicates the total number of the training parameters within the model, and how its complexity scales.

By measuring the performance of these four models against each other, we have the results for the AUC-ROC score in Figure 2.6.

The results of this model will be directly compared to the results of the performance of the model described in this thesis.

Multilingual deep neural networks [16] are another avenue of research when trying to classify music genres. These have extensively been used in low resource automatic speech recognition (ASR). In order to gain a better performance on classifiers trained without an abundance of data, ASR uses multi-linugal data to match the performance. When translated to the music information retrieval domain, this publication utilizes the nearest neighbor algorithm to find the most similar resource data from a large similar database. While [3] uses the Million Song Database (which is used by this thesis, this publication [16] trains on the GTZAN [5] and the ISMIR 2014 Genre Database[6]. The peak accuracy when using three layers each with 1024 hidden nodes, is stated too bee $93.4 \%$ according to this publication. While this is a very interesting research topic, in a scenario where there are gigantic amounts 
of data, personalizing recommender systems with limited data neural networks may decrease the performance of the core classifier.

The final publication [17] examined within Section 3 combines the techniques described in the traditional classification techniques for feature extraction. It differentiates the features into spectral and temporal features, where spectral features represent the characteristics of music in a relatively short period of time and therefore reveal timbre and tonal characteristics - Temporal characteristics, define the long term dynamics of the signal and is not always mutually exclusive to spectral features. The input to this preprocessing are 30 s clips of each of the tracks.

In order to calculate the spectral and the temporal features, the track is transformed into cepstrogram domain, which is the harmonic decomposition of a logarithmic magnitude spectrum using the inverse discrete Fourier Transform (DFT). A cepstogram is calculated from a magnitude spectogram using the equation below:

$$
\left.C_{\mathrm{i}}(q, t)=\frac{1}{N} \sum_{f=0}^{N-1} \ln \left(X_{i}\right)(f, t)+\epsilon\right) \exp \left(\frac{2 \pi q f j}{N}\right)
$$

Further processing is performed in order to input into the deep neural network for temporal feature extraction. A random forest was used as the classifier, resulting in an accuracy of $72.6 \%$ when using only the temporal features, and $78.2 \%$ while using the spectral features. Combining the two sets of features resulted in an accuracy of $85.0 \%$. Some of the ideas of extracting spectral features from the audio track itself, using a deep neural network and 
supplying it to a classifier, have been adopted within this thesis extensively. Within the model of this thesis, care has been taken to supply both spectral and temporal features have been supplied to the classifier.

\subsection{Hierarchical Attention Networks}

The driving force behind behind this thesis is the innovative document classification model called hierarchical attention networks (HAN) [18]. The hierarchical structure of the model mirrors the hierarchical structure of the documents, where it has two attention mechanisms applied at word and sentence level. The authors of the publication noticed that different words and sentences within the documents are deferentially informative, especially dependant on the context. The parallels between the hierarchical structure of a document and the hierarchical structure of an audio track is what inspired the adaptation of a HAN for the task of music genre classification.

The overall architecture of the HAN is described by Figure 2.7. The sequence encoders use a GRU that computes a new state $h_{t}$ as a linear interpolation between the previous state $h_{t-1}$ and incoming new sequence information. The candidate state $\tilde{h}_{t}$ is computed in a similar way to an RNN, where it goes through a tanh activation and uses information from $h_{t-1}$. The update gate $z_{t}$ decides how much old information and new information is added, while the reset gate contributes to how much the past state contributes to the candidate state. 
Most document classifiers focus on turning a document with L sentences (each denoted by $s_{i}$ ) and each sentence containing $T_{i}$ words. $w_{i t}$ represents the the $t^{\text {th }}$ word in the $i^{\text {th }}$ sentence into a vector representation. the publication on HANs also aims on doing so, but synthesizes the document vector by using the word and the sentence encoders. The word encoder comprises of a bi-directional GRU that using an embedding $\left(W_{e}, x_{i j}=W_{e} w_{i j}\right)$ matrix that has been inputted to the encoder. The forward and the backward hidden states of the bi-direction GRU aree then concatenated to form the final hidden state. Due to the fact that not all words contribute equally to the representation of the sentence meaning, an attention network is used to create the sentence vector. The hidden state of the word the word annotation $h_{i t}$ goes through a one layer fully connected layer to geet the hidden representation $u_{i t}$ which is then compared with the word level context vector $u_{w}$ to get the normalized importance weight $a_{i t}$ (through a softmax function) to compute the sentence vector $s_{i}$ which is the weighted sum of all of the word annotations. The word context vector is randomly initialized and then jointly learnt through the training process.

With the sentence vectors $s_{i}$ in place, another bidirectional GRU encodes the sentences to as a concatenation of a forward and backward read of the sentence vectors. Similar to the word based attention network, a document vector $v$ is calculated by a weighted sum of all the sentence vectors in relation to the sentence level context vector. Performing softmax on the document level vector gives us our classification. 


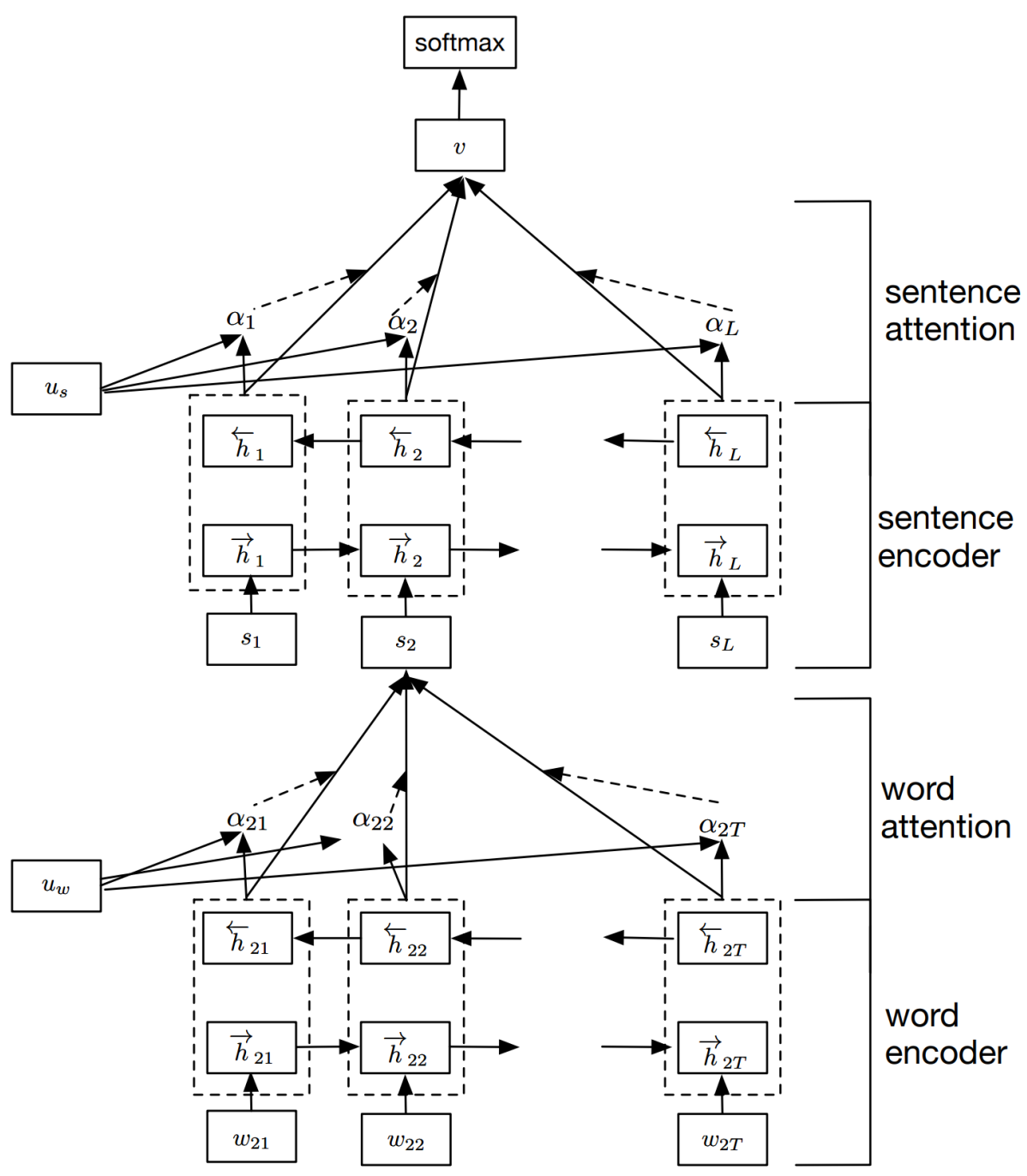

Figure 2.7: A heirarchical attention network. 


\section{Chapter 3}

\section{Proposed Method}

The overall architecture of the system follows the process as described by Figure 3.1. The input to the system while training and evaluating is an audio file. The input goes through the feature extraction, where multiple features are extracted, converted into their symbolic text form and then appended into a single text representation. This is then converted into a word embedding representation that is the input to the HAN, which proceeds to classify it. Some of the ideas of extracting spectral features from the audio track itself, using a deep neural network and supplying it to a classifier, as explained in [16] have been adopted within this thesis extensively. Using inspiration from the aforementioned publication, thirty second clips from the start of every track are used as input into the proposed method.

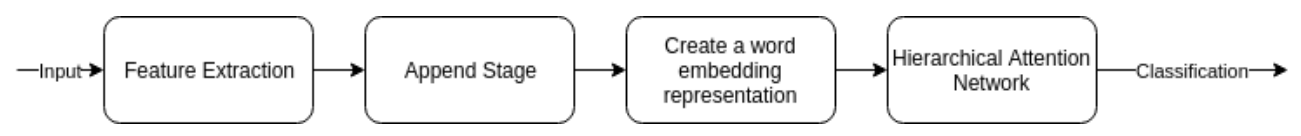

Figure 3.1: The outline of the architecture being proposed by this thesis. 


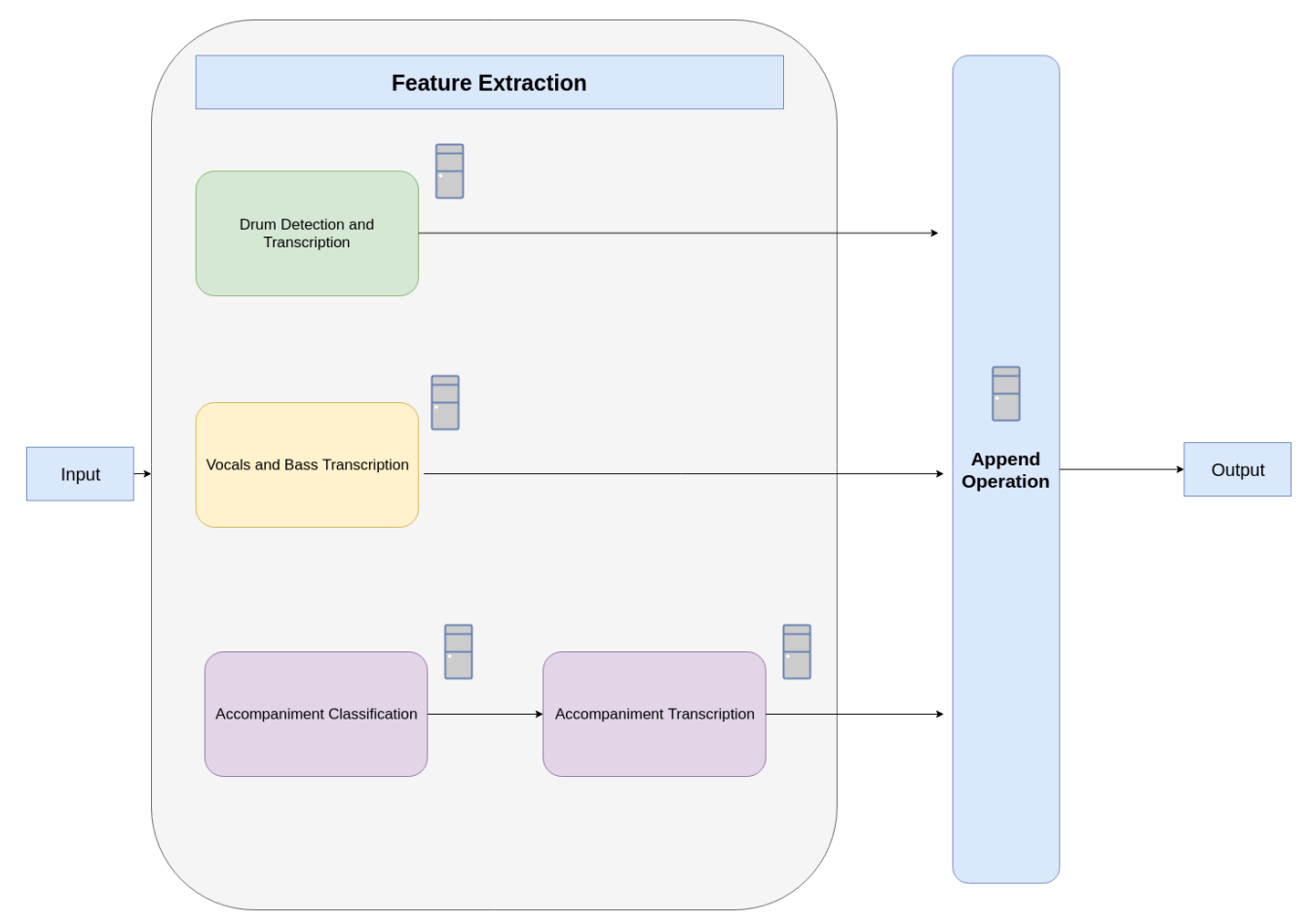

Figure 3.2: The feature extraction architecture being proposed by this thesis.

\subsection{Feature Extraction}

Most of the techniques that were adopted to extract features are adapted from novel academic publications that use deep learning. The architecture for the preprocessing(feature extraction) is given in Figure 3.2.

\section{Extraction and Symbolic Conversion of Drums}

Within the preprocessing, we first approach the extraction and symbolic conversion of the

drums. In order to do this, we have used ADT[19]. Automatic Drum Transcription softwares attempt to generate symbolic musical notation of percussive instruments in music recordings 


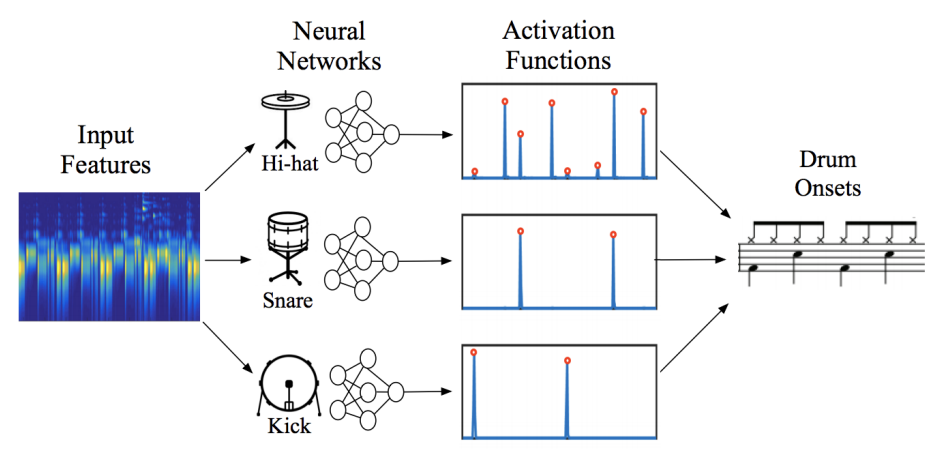

Figure 3.3: Drum Transcription Neural Network

through neural networks. A bi-directional recurrent neural network for the detection of percussive onsets for the specified drum classes (hi-hats, kicks and snares) or the combinations of, has been implemented by this thesis. For each instrument, the neural network is identical, consisting of three layers of 50 neurons. The performance measure used is categorical cross entropy and softmax, with a precision of 0.939 , recall of 0.934 and F1 measure of 0.929 . This technology was chosen for its accuracy. An example of a transcription is given below:

$$
\begin{aligned}
& 0.1625 \mathrm{HH} \\
& 0.3599 \mathrm{HH} \\
& 0.3831 \mathrm{KD} \\
& 0.5689 \mathrm{SD}
\end{aligned}
$$

As it can be seen, the specific time at which (in seconds) has been given in the transcribed file. $H H$ represents a hi-hat, $K D$ represents a kick drum, while $S D$ represents a snare drum. It is expected that this should be enough information for a classifier to learn about the time 
signature, the style of drumming (which often map to a particular genre) and the tempo, and thus be able to differentiate between genres better, as most western based genres comprise of unique drumming styles. With the representation of the drums and their timings intact, phrases can intelligently be determined from the data, and each of these phrases

\section{Separation of Different Tracks}

Chandna, et al. [20] have proposed a low latency monoaural source separation framework using a convolutional neural networks to estimate the time-frequency soft masks which are applied onto the audio input (the original track in our case), and can currently separate voice, drums, bass and accompaniment from the source. The encoding stage of this model, contains two convolution layers and a Multi-Layer Perceptron(MLP, which is also known as a fully connected layer). The decoding stage of this model contain a fully connected layer with ReLU activation. Finally deconvolution layers give us the output.

This model is essential to the proposed solution, as this thesis is trying to look at the relation between different instruments within the audio track. Separating the different tracks would give the classifier a unique insight into the tracks that are being classified. This is best exemplified in a genre such as jazz. During the change of the chord within the chord progression repetitions, it is often found that the bass playing a note that is not in the scale but is one semitone higher or lower than the root note of the next chord. This note, often called the chromatic approach note is played a quarter of note before the onset of the next 


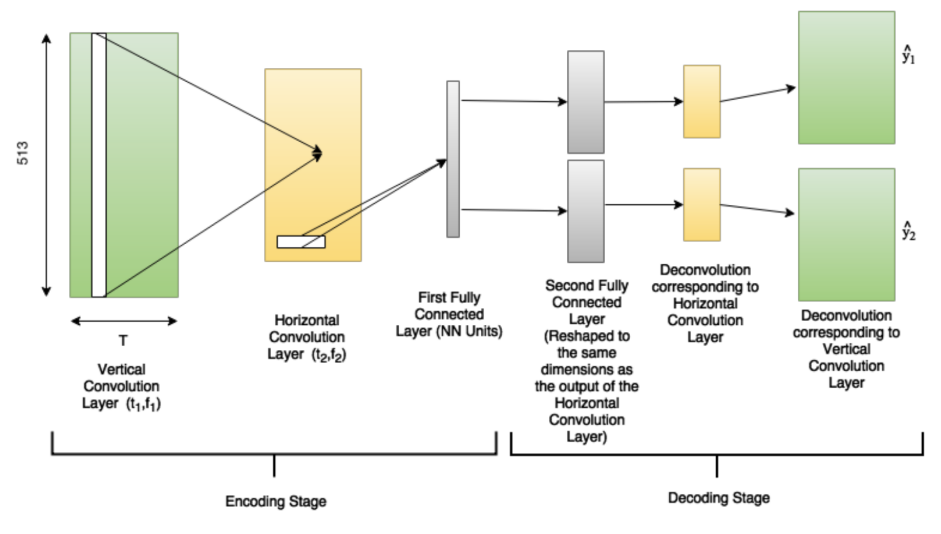

Figure 3.4: Architecture of the Track Separation Neural Network

chord. The proposed solution was engineered in a manner where the classifier should be able to learn this common technique within jazz and other relevant techniques in other genres.

\section{Classification of Accompanying Track}

Once the different instruments have been separated, the accompaniment is classified, in order to gain more insight about the genre. Due to the evolution of western music, a particular instrument has played a very vital role in the development of a specific genre. This is often an instrument that encompasses a large frequency of playable notes (therefore not the bass or the drums). some examples of this is the use and emphasis of a distorted lead guitar in rock music since its conception in the late 1950s, and the embellishment and prominence of different kind of saxophones and wind instruments within the various sub genres of jazz music. Due to the creation of electronic amplification and modification of the tonality and 
timbre of a guitar, the statistic variance of the sound of a guitar would be greater than that of the variance of the tonality of different wind instruments within jazz. It is hypothesized that the accompaniment classification will be useful within the proposed solution for this reason.

In order to identify the particular lead or instrument that is accompanying our audio track, we will have to classify it before sending this information to the final classifier. With the help of Deep Instrument Heroku Deep Learning Application [21], this can be achieved. The application computes reassigned chromagrams (log-frequency log-amplitude spectrograms with bins aligned aligned to pitches) from the audio. The output for each audio clip is a matrix (time frames, chroma bins). Together they are in a matrix of shape (data points, time frames, chroma bins). The output of this program is single word (sometimes a double barreled word, such as alto-saxophone), that is appended to the start of the transcription of the accompaniment.

\subsection{Conversion of Melodic Data into Symbolic Data}

The proposed solution extracts the following values from the respective melodic tracks (vocals, bass or accompaniment). A sample output of the values from a clip of a MIDI track has been displayed below. This is in fact the very beginning of Mary had a Little Lamb.

231 On $\operatorname{ch}=1 \mathrm{n}=64 \mathrm{v}=0$

256 On $\operatorname{ch}=1 \mathrm{n}=62 \mathrm{v}=72$ 


$$
\begin{aligned}
& 487 \text { On } \operatorname{ch}=1 \mathrm{n}=62 \mathrm{v}=0 \\
& 512 \text { On } \mathrm{ch}=1 \mathrm{n}=60 \mathrm{v}=71 \\
& 743 \text { On } \mathrm{ch}=1 \mathrm{n}=60 \mathrm{v}=0 \\
& 768 \text { On } \mathrm{ch}=1 \mathrm{n}=62 \mathrm{v}=79 \\
& 974 \text { On } \mathrm{ch}=1 \mathrm{n}=55 \mathrm{v}=0 \\
& 999 \text { On } \mathrm{ch}=1 \mathrm{n}=62 \mathrm{v}=0
\end{aligned}
$$

There are five different components to the MIDI output that are explained below. It is also mentioned whether they are discarded or processed as output.

1. Timestamp: This is the time stamp of the start of the midi note in terms of microseconds. The delta information was ignored, as we are creating sentences from the starting point of each and every key.

2. State: The note is either on or off. This is a feature that has been ported over from the MIDI files that are outputted by digital and software synthesizers.

3. Channel; While recording multiple instruments, or a single instrument containing a rack of other instruments, the MIDI is categorized by its channel. This is ignored, as we are artificially converting our tracks into MIDIs, and therefore will only contain a single MIDI channel at all times.

4. Note: Represented by $n$, this is usually an integer value that ranges between 0 and 127. This value indicates the pitch of the note. A high pitch value indicates that it is 
of a high octave.

5. Velocity: In order to express human inflection in the music, some notes are played with more intensity that others. This is best represented by the velocity which varies between 0 and 127. Since the playing style of a lot of human beings varies, and emphasis is placed on notes in different order this field was effectively ignored. However, some genres like Funk, do emphasize on a very intense and rigid first note, every couple of beats.

\subsection{Intelligent Phrase Separation and Append}

\section{Operator}

Multiple techniques were used in order to create the final document that will be classified by the proposed solution. The differences in various techniques and how they have utilized the preprocessed features has been further expanded upon in the Section IV of this publication. Some of the techniques for further processing the preprocessed features have been defined below.

The MIDI transcriptions are converted into text through by a translation where the note and its octave are appended together. For example, the MIDI entry which contains $n=48$, is represented by the text "C3". The next entry which could be in the form of "768 On ch $=1 n=53 v=79$ " is represented by the note "E3". At this point the combined 
text would be the two notes combined together separated by a space, thus emphasizing each word as a note.

If the velocity of the note were to be considered. it would be appended along with the value of the velocity of the note. The previous mentioned progression of C3 followed by E3 could be represented by something similar to "C345 E379".

While different combinations of the constituent elements, separated as paragraphs were used to inform us about the performance of the system, the overarching order always remained the same, as listed below. This order has been selected due to the frequency of usage of each of these features in previous texts that have been encountered in the literature survey.

1. Bass (always used)

2. Vocals

3. Drums

4. Accompaniment

5. Classification of the Accompaniment

Each of these were transcribed using the techniques above, except for the classification of the accompaniment, which was left as a word that described the instrument. For example, on a rock song, "guitar" would often be the classification of the accompaniment, leading to the last text within the appended text document to be "guitar". When the drums were 
appended, their onsets were rounded to three significant figures and multiplied by a 100 . The entirety of the text, often referred to as a document within NLP is referred to as a transcription within this publication.

By using only these transcriptions, which are high level music content description, automatically created by a machine, we are inputting data that is readable by experts in the music industry opening up many avenues of collaboration in order to improve the performance of systems in question.

\subsection{Creating the word Embeddings}

While a lot of pre-trained embeddings are available for public usage, due to the novelty of this problem and the nature of the synthesis of two different academic disciplines, a custom skip-gram embedding was trained for this problem. Skip gram was chosen since it has been hypothesized that skip gram functions well with a small amount of training data.

In order to train the custom embedding, the input dimensions (size of the vocabulary) of the text was calculated by counting the unique number of words within all the example transcriptions $\left(U_{i}\right)$ which weren't part of the natural set of a given training process $\left(D_{i}\right)$. The natural set can be calculated as the combination of all the different notes and their octaves and their MIDI onsets plus the combinations of the drums and their possible onsets. This has been shown in the equation below for the $\mathrm{S}$ (input size) for the situation where our input transcription is the combination of the vocal, bass and accompaniment transcription 
(without velocity) along with the class of the accompaniment.

$$
\begin{gathered}
S=U_{i}+D_{i} \\
S=1+3 \times 128=385
\end{gathered}
$$

In this particular case, since there are three different instruments, each with 128 possible notes and a single word for the accompaniment class, the input vocabulary dimensions equals 385. The output dimensions are consistently set to be 128. It is hypothesized that this leads to maximum efficiency, while also being large enough to capture the semantics of the text. The length of the input transcriptions is calculated by finding out the maximum length of all the transcriptions and the rest are padded with the note "C-1" in order to get an equal length among all the input transcriptions.

\subsection{Calculating Sentence Length}

Assuming that we have the tempo, we can now calculate the optimal sentence length that represents a phrase within a particular transcription. It is important that we have a good representation of the sentence within a transcription, as the advantage of using a HAN would be lost, unless we can represent the structure of the document(transcription) as words and sentences, which in our case are notes and phrases. ADT which is responsible for the transcription of the drums, often gives us the tempo of the track (the tempo of the track 
can be represented by $T$ ), can be used within this calculation. If the length of the track is represented by $l_{t}$ in seconds, we can use the raw MIDI transcriptions to calculate the average number of notes per phrase $\left(N_{p}\right)$, as shown in the equation below. First wee calculate the average number of phrases per minute $(T / 32)$. This result is further divided by 60 to give us the number of phrases per second $\left(N_{\text {phrases }} / \mathrm{sec}\right)$. We can calculate the total number of phrases within the track and therefore the average number of notes per phrase, as shown below (where $w_{i}$ is the $i_{t h}$ note in the transcription).

$$
\begin{gathered}
N_{\text {totalphrases }}=\left(N_{\text {phrases }} / s\right) \times l_{t} \\
N_{p}=\frac{\sum_{i} w_{i}}{N_{\text {totalphrases }}}
\end{gathered}
$$

This gives us an optimal size of the sentence when training and evaluating the model.

\subsection{Classification Pipeline}

The classification pipeline is adapted from the HAN publication, however, with minor design modifications for performance and convenience.

L2 Regularization and Dropout were not used as empirically within the scale of this project, over fitting does not seem to be a problem. However, this could be an avenue of further research, were the scale of the data and classification problem increase in size.

While the performance of a GRU is almost on par with an LSTM, the proposed solution was implemented on a system with enough computational power available to incorporate an 


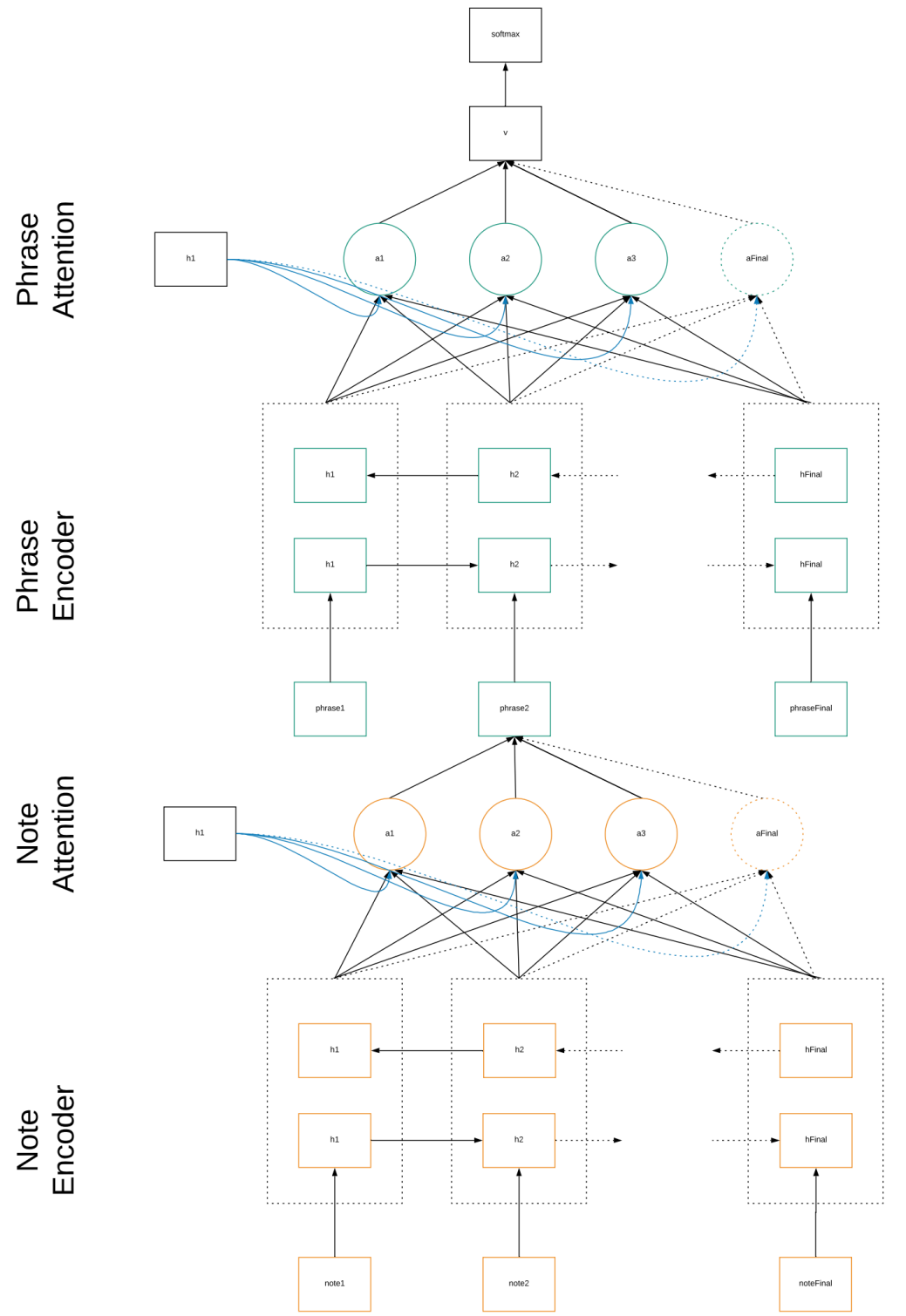

Figure 3.5: Modified HAN that's being used for the classification pipeline of this publication. 
LSTM, instead of a GRU within this particular project. Therefore the RNN unit used for the classification model was an LSTM instead of an RNN. Since the number of RNN units in both the note and phrases stages of the HAN are the same an arbitrary value of 200 was chosen for both the number of RNN units and the dense layer. The complete architecture of this proposed classification pipeline is shown in Figure 3.5. The structure of the HAN is almost identical to the one that was described in Chapter 2.

Softmax was used for activation, as we are not considering this as a multi class classification problem and the classes are therefore considered to be mutually exclusive. Categorical cross entropy was used for the loss function over Mean Squared Error. Adam was chosen as the optimizer. 


\begin{tabular}{|lc||lc|}
\hline GTZAN & & ISMIR & \\
\hline genre & tracks & genre & tracks(train/test) \\
\hline classical & 100 & Classical & $320 / 320$ \\
jazz & 100 & Electronic & $115 / 114$ \\
blues & 100 & Jazz/Blue & $26 / 26$ \\
metal & 100 & Metal/Punk & $45 / 45$ \\
pop & 100 & Rock/Pop & $101 / 102$ \\
rock & 100 & World & $122 / 122$ \\
country & 100 & & \\
disco & 100 & & \\
hiphop & 100 & & \\
reggae & 100 & & \\
\hline total & 1000 & total & \\
\hline
\end{tabular}

Table 4.1: Breakdown of the number of tracks per genre on the GTZAN[5] and ISMIR[6] datasets 


\section{Chapter 4}

\section{Implementation Deatils}

\subsection{Dataset}

Most of the publications reviewed in Chapter 2, and related publications within the music information retrieval communities incorporate the use of the Million Song Dataset [22]. The Million Song Dataset (MSD) is a free collection of over a million contemporary tracks. Some of the auxiliary datasets that have been provided on the Million Song Dataset are the MAGD dataset which includes multiple genre labels and references to MSD. Other datasets are compared within Figure 4.1 for reference. It is clear that for a proposed solution that is aiming to stay relevant and focused on solving problems within the industry as of right now, employing MSD, as the dataset is the clear choice.

In order to engineer the correct solution for the massive amount of data, multiple solutions were employed. One of these solutions is the use of MongoDB [23]. MongodB uses a 
selection of three different genres and continues to randomly select a hundred entries from each genre through the use of an aggregation pipeline. After this stage, each of the entries are searched for on YouTube and downloaded as .mp3 data automatically. Due to the potential of parallelization during this phase of the data preparation, this task was distributed among the multiple workers in the spark cluster (which is talked about more in detail within the Distributed Learning section). The data from each of these tracks is stored within an RDD, which functions as input to the next section, preprocessing.

\subsection{Preprocessing}

Most of the programs written within the preprocessing section were adapted from existing publications which have been implemented in TensorFlow on websites such as Github. Some of these needed modification in order to integrate with the overall pipeline of the proposed method. one major modification which most of the software went through was an integration into a tensoronSpark application which allows TensorFlow to run on a Spark/Hadoop Cluster.

\subsection{Testing and deep learning frameworks}

All the different preprocessing units were implemented in TensorFlow, while the modified HAN at the end was implemented in Keras. 


\begin{tabular}{|l|l|l|}
\hline Node Name & GPU(s) & Testing/Training \\
\hline Master & $2 \times$ NVIDIA GeForce RTX 2080 & Testing + Training \\
\hline Slave 01 & NVIDIA GeForce GTX 1070 & Training \\
\hline Slave 02 & NVIDIA GeForce GTX 1070 & Training \\
\hline Slave 03 & NVIDIA GeForce GTX 1070 & Training \\
\hline Slave 04 & NVIDIA GeForce GTX 1070 & Training \\
\hline
\end{tabular}

Table 4.2: The specifications of the experimental setup. it must be noted that testing took place on a single computer, which was the master and also the system with the largest GPU memory available.

\subsection{Distributed Learning}

In order to deal with the magnitude of the data, the proposed solution's pipeline was programmed over multiple nodes in a cluster manner. This is further expanded on, within the first subsection detailing the Spark implementation of the solution. The input to this architecture is the audio track in either a .mp3 or a .wav format. The output is the classification of the particular track.

\section{Spark Implementation}

Due to the amount of memory and processing power needed to compute and use the Million Song Dataset, a distributed framework was decided upon. Due to the popularity of Spark [24], and its extensive uses within the academic and professional communities, Spark was used in order to distribute the computations and training within both the preprocessing pipeline and the final classifier. The network architecture is shown below with the master and the slave nodes working together in order to distribute computation. This network over 
a Virtual Private Network(VPN), where the master was the responsible for the division of the work among the cluster nodes which are each named slaveX, where $\mathrm{X}$ is substituted by the numerical assignment of said computer. In order to achieve distributed computing, multiple frameworks have been used, each of which have been described below.

\section{PySpark}

PySpark [25] is the framework that exposes Spark in Python. Since most of the deep neural networks that are used within this publication are written in Python, it is imperative that the framework that distributes these networks computationally, is written in the same language. The songs that are selected for the training of the machine learning models, are downloaded from YouTube parallely and stored in a Resilient Distributed Dataset(RDD). This data is used to create a dataframe that persists till the final classification, where the metadata is converted into an RDD again.

\section{Elephas}

Elephas[26] is an extension of Keras that aims to distribute Keras training over a distributed Spark cluster. While quite effective at distributing the computations, Elephas lacked many features that had to be implemented in order to properly analyze the performance of the model over a distributed cluster. Efforts taken by the author in order to accomplish this, has been discussed in this section along with an overview of Elephas. 


\section{Chapter 5}

\section{Results}

The first set of results are from looking at different combos of different genres. With a threshold of confidence set to be above 0.5 , the results combos of three different genres and their results has been shown in Table 5. In order to come up with these combinations, some musicological knowledge has been used for the best selection in terms of contemporary popularity and a lack of redundancy. Due to the fact that many genres are derived from each other, combinations of genres with a recent common root or combinations where one was derived from each other, have been avoided. However, exceptions such as Pop Rock, RnB, Blues exist where disco the genres share a common ancestor funk, but have either evolved to sound very different or have not evolved past their initial style. The results in the table were compiled by appending the transcription of the bass, vocals and the accompaniment, without the velocities.

Using the combination of hip hop, jazz, rock, different compositions of transcriptions 


\begin{tabular}{|l|l|}
\hline Genres & F1 Score \\
\hline \{Reggae, Country, Pop Rock & 0.85 \\
\hline \{Country, Jazz, Rap & 0.87 \\
\hline \{Latin, Jazz, Blues & 0.76 \\
\hline PPop Rock, RnB, Blues & 0.84 \\
\hline PPop Rock, Jazz, Rap & 0.92 \\
\hline
\end{tabular}

Table 5.1: The performance of different combinations of genres and their respective F1 score rounded to two significant figures.

\begin{tabular}{|l|l|l|}
\hline \multirow{3}{*}{ Without Velocities } & bass + vocals & 0.85 \\
& bass + accompaniment & 0.87 \\
& bass + vocals + accompaniment & 0.92 \\
& bass + vocals + drums + accompaniment & 0.78 \\
& bass + vocals + drums + accompaniment + accompaniment classification & 0.81 \\
\hline \multirow{3}{*}{ With Velocities } & bass + vocals & 0.81 \\
& bass + accompaniment & 0.71 \\
& bass + vocals + accompaniment & 0.83 \\
& bass + vocals + drums + accompaniment & 0.78 \\
& bass + vocals + drums + accompaniment + accompaniment classification & 0.79 \\
\hline
\end{tabular}

Table 5.2: The performance of the proposed solution with different combinations of preprocessed features

were used to examine the F1 score. These have been detailed in Table 5. As it can be seen the combination of the bass, vocals and accompaniment without velocities gives us the best performance. The best of these scores are either equal to better than the peak performance of $[16]$.

It can be hypothesized that given the nature of the solution architecture, dealing with augmented velocities led to worse performance. This could be due to the representation of context becoming a lot more complex during the embedding solution, or due to the size of the vocabulary. Adding the drums leads to a similar increase in the size of the vocabulary, and therefore leading to less useful embedding. 
An Area Under the Curve - Receiver Operating Curve has been plotted in Figure 5.1. ROC curves are a great way of visualizing the trade-off between sensitivity and specificity. Due to the melodic similarity between most pop rock tracks, it is not surprising that this is genre shows the best performance among the three that are being considered. Jazz on the other hand is one of the oldest surviving genres from the United States of America and is often reputed for its melodic complexity. Therefore we can hypothesize that genres with lesser melodic complexity fare better with the proposed solution of this publication. The success of this system could be due to the hierarchical structure of the modified HAN parsing through the high level music content description, which was informed by the knowledge of musicology. 


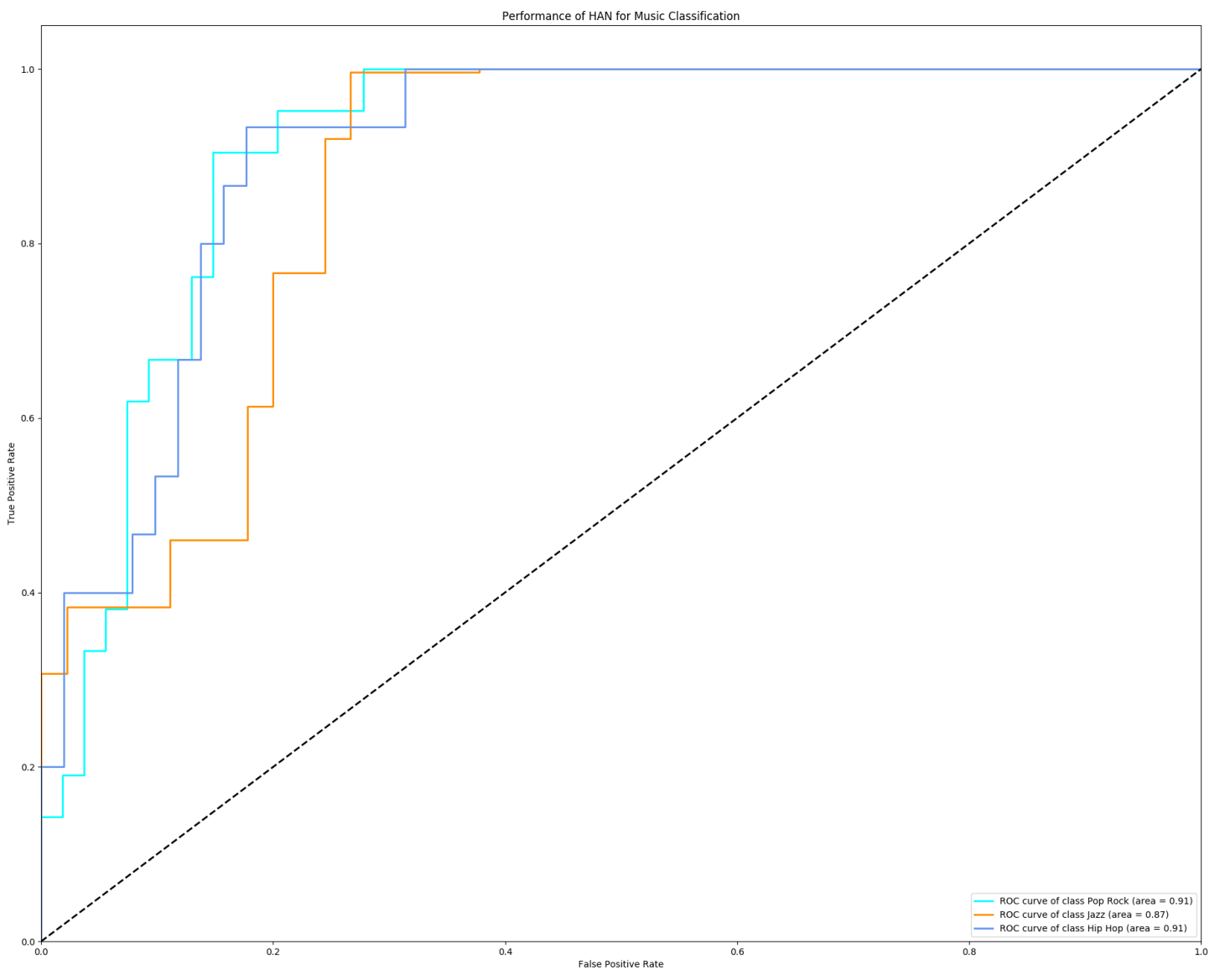

Figure 5.1: The AUC ROC graph as per the different genres. 


\section{Chapter 6}

\section{Conclusion}

\subsection{Conclusion}

We can conclude that our research has performed with a satisfiable AUC-ROC of 91\%, when compared with earlier publications (Choi et al. [3] claim a performance of 0.865 with a CRNN at $3 \times 10^{6}$ number of parameters) This publication introduces a novel method of converting a musical piece into its musicological symbolic notation and using a Hierarchical Attention Network for genre classification. The use of distributed computed accelerated the training of the system, and increased the depth of the investigation. Some of the future directions this research could take is the incorporation of self-collected datasets. Multi-label classification in another avenue that requires far more research. Due to the prevalence of digitized music, musicians are deriving inspiration from various genres and combining them in creative ways. Therefore, multi-label classification would be quite useful as this increases 
the usefulness of recommender systems significantly.

\subsection{Future Works}

There are many aveenues for future work within this thesis. The first avenue would be to investigate other classification methods such as convolutional neural networks for the task of text classification with the particular preprocessed features from this publication. In recent years, CNNs have been enjoying a lot more success within the natural language processing field. This could also possibly help with the processing of the accompaniment classification and the drum onsets, which seem to have not been too useful with an RNN.

Furthermore, intelligent clipping of the track itself could be performed. Right now, only the first thirty seconds of the track are used as the input to preprocessing. There are methods that exist that can identify the difference between various sections of the track, such as the bridge and the chorus. Intelligently sampling this data, or using the entire track as an input could lead to the proposed model learning a lot more info about the track. Therefore, it can be hypothesized that the performance of the model could improve significantly.

Finally, using multi-label data could be beneficial to the industrial applicability of this project. Realistically, in the twenty first century most genres have very blurred borders and the classification of tracks is very subjective. Other genres like pop can be studied too, which has a very dynamic evolving sound only characterized by it's mastering (the volumes of the different instruments) and the frequency spectrum that these instruments occupy. 


\section{Bibliography}

[1] D. Britz, Attention and Memory in Deep Learning and NLP. Wild ML.

[2] N. Donges, Recurrent Neural Networks and LSTM. Towards Data Science.

[3] K. Choi, G. Fazekas, M. Sandler, and K. Cho, "Convolutional recurrent neural networks for music classification," in 2017 IEEE International Conference on Acoustics, Speech and Signal Processing (ICASSP), pp. 2392-2396, IEEE, 2017.

[4] E. Zheng, M. Moh, and T.-S. Moh, "Music genre classification: A n-gram based musicological approach," in 2017 IEEE 7th International Advance Computing Conference (IACC), pp. 671-677, IEEE, 2017.

[5] B. L. Sturm, "An analysis of the gtzan music genre dataset," in Proceedings of the second international ACM workshop on Music information retrieval with user-centered and multimodal strategies, pp. 7-12, ACM, 2012.

[6] P. Cano, E. Gómez Gutiérrez, F. Gouyon, P. Herrera Boyer, M. Koppenberger, B. S. Ong, X. Serra, S. Streich, and N. Wack, "Ismir 2004 audio description contest," 2006.

[7] M. Li and R. Sleep, "Melody classification using a similarity metric based on kolmogorov complexity," Sound and Music Computing, vol. 2012, 2004.

[8] A. Kotsifakos, E. E. Kotsifakos, P. Papapetrou, and V. Athitsos, "Genre classification of symbolic music with smbgt," in Proceedings of the 6th international conference on PErvasive technologies related to assistive environments, p. 44, ACM, 2013.

[9] C. McKay and I. Fujinaga, "Automatic genre classification using large high-level musical feature sets.," in ISMIR, vol. 2004, pp. 525-530, 2004.

[10] T. Mikolov, I. Sutskever, K. Chen, G. S. Corrado, and J. Dean, "Distributed representations of words and phrases and their compositionality," in Advances in neural information processing systems, pp. 3111-3119, 2013.

[11] A. Waibel, T. Hanazawa, G. Hinton, K. Shikano, and K. J. Lang, "Phoneme recognition using time-delay neural networks," Backpropagation: Theory, Architectures and Applications, pp. 35-61, 1995. 
[12] N. Kalchbrenner, E. Grefenstette, and P. Blunsom, "A convolutional neural network for modelling sentences," arXiv preprint arXiv:1404.2188, 2014.

[13] T. L. Li, A. B. Chan, and A. H. Chun, "Automatic musical pattern feature extraction using convolutional neural network," Genre, vol. 10, p. 1x1, 2010.

[14] J. Wülfing and M. A. Riedmiller, "Unsupervised learning of local features for music classification.," in ISMIR, pp. 139-144, 2012.

[15] J. Schlüter, "Learning to pinpoint singing voice from weakly labeled examples.," in ISMIR, pp. 44-50, 2016.

[16] J. Dai, W. Liu, C. Ni, L. Dong, and H. Yang, "multilingual deep neural network for music genre classification," in Sixteenth Annual Conference of the International Speech Communication Association, 2015.

[17] I.-Y. Jeong and K. Lee, "Learning temporal features using a deep neural network and its application to music genre classification.," in Ismir, pp. 434-440, 2016.

[18] Z. Yang, D. Yang, C. Dyer, X. He, A. Smola, and E. Hovy, "Hierarchical attention networks for document classification," in Proceedings of the 2016 Conference of the North American Chapter of the Association for Computational Linguistics: Human Language Technologies, pp. 1480-1489, 2016.

[19] C. Southall, R. Stables, and J. Hockman, "Automatic drum transcription using bidirectional recurrent neural networks.," in ISMIR, pp. 591-597, 2016.

[20] P. Chandna, M. Miron, J. Janer, and E. Gómez, "Monoaural audio source separation using deep convolutional neural networks," in International conference on latent variable analysis and signal separation, pp. 258-266, Springer, 2017.

[21] bzameknic, Musical Instrument Classification. Github.

[22] T. Bertin-Mahieux, D. P. Ellis, B. Whitman, and P. Lamere, "The million song dataset," 2011.

[23] K. Chodorow, MongoDB: the definitive guide: powerful and scalable data storage. " O’Reilly Media, Inc.", 2013.

[24] M. Zaharia, M. Chowdhury, M. J. Franklin, S. Shenker, and I. Stoica, "Spark: Cluster computing with working sets.," HotCloud, vol. 10, no. 10-10, p. 95, 2010.

[25] J. Rosen and S. Owen, "pyspark internals," Disponivel em: https://cwiki. apache. org/confluence/display/SPARK/PySpark+ Internals. Acessado em, vol. 14, no. 12, p. 2017, 2016. 
[26] M. Pumperla, "Elephas: Distributed deep learning with keras \& spark." 